\title{
An Effectively Modified Firefly Algorithm for Economic Load Dispatch Problem
}

\author{
Nguyen Trung Thang ${ }^{* 1}$, Nguyen Duy Phuong ${ }^{2}$, Pham Van Thanh ${ }^{3}$, Chiem Trong Hien ${ }^{4}$ \\ ${ }^{1}$ Power System Optimization Research Group, Faculty of Electrical and Electronics Engineering, \\ Ton Duc Thang University, Ho Chi Minh City, Vietnam \\ ${ }^{2}$ Faculty of Electronics-Telecommunications, Saigon University, Ho Chi Minh City, Vietnam \\ ${ }^{3}$ Faculty of Electrical Engineering and Computer Science, Technical University of Ostrava, Czech \\ ${ }^{4}$ Faculty of Electrical and Electronic Technology, Ho Chi Minh City University of Food Industry, Ho Chi \\ Minh City, Vietnam \\ *Corresponding author, e-mail: nguyentrungthang@tdtu.edu.vn
}

\begin{abstract}
This paper proposes an effectively modified firefly algorithm (EMFA) for searching optimal solution of economic load dispatch (ELD) problem. The proposed method is developed by improving the procedure of new solution generation of conventional firefly algorithm (FA). The performance of EMFA is compared to FA variants and other existing methods by testing on four different systems with different types of objective function and constraints. The comparison indicates that the proposed method can reach better optimal solutions than other FA variants and most other existing methods with lower population and lower maximum iteration. As a result, it can lead to a conclusion that the proposed method is potential for ELD problem.
\end{abstract}

Keywords: firefly algorithm, economic load dispatch, single fuel, multi fuels

Copyright (c) 2018 Universitas Ahmad Dahlan. All rights reserved.

\section{Introduction}

In ELD problem, the thermal units can use either only one fuel type or multi fuel types and their fuel cost function form is dependent on the option of use. In case that only one fuel type is used for purpose of electricity generation, fuel cost function is approximately represented as a second order equation [1-3]. Besides, when multi fuel types including coal, natural gas and oil can be used for a unit, fuel cost function is mathematically formulated as a segmentedpiecewise quadratic function [4-8].

Traditionally, deterministic methods have been used for solving the ELD problem, such as Lagrange relaxation [9] and Hopfield network based methods [10]. The methods have the same potential ability such as short execution time, few parameters and one optimal solution. However, these methods also own the same disadvantages such as limited applicability for problems with complicated constraints and less efficiency for large scale problem. To avoid overlapping such drawbacks of deterministic methods, many meta heuristic approaches have been constructed to deal with the ELD problem such as teaching and learning based algorithm (TLBA) [1], cuckoo search algorithm (CSA) [2-3, 11-12], hybrid real coded genetic algorithm (HRCGA) [4], differential evolution (DE) [5], genetic Algorithm (GA) [5], particel swarm optimization (PSO) [5], improved evolutionary programming (IEP) [6], clonal algorithm (CA) [7], hybrid integer coded differential evolution (HICDE)[8], bee colony optimization (BCO) [13], biogeography-based optimization (BBO) [14], chaotic jaya algorithm (CJA) [15], firefly algorithm (FA) [16],memetic firefly algorithm (MFA) [16], FA with adaptive parameter a (ASPFA) [16], modified firefly algorithm ( $\alpha-M F A)$ [17], seeker optimization algorithm (SOA) [18], krill herd algorithm (KHA) without using genetic operation (KHA1) [19], KHA using crossover operation (KHA2) [19], KHA using mutation operation (KHA3) [19] and KHA using crossover operation and mutation operation (KHA4) [19]. These methods have shown their powerful search ability via testing on different problems with different conditions such as different constraints related to thermal units and different constraints associate with power systems.

Firefly algorithm is also population based meta-heuristic algorithm like GA, PSO and other methods. The method was constructed by Yang in 2008 for solving optimization 
problems [20]. The method has been demonstrated effectively but its effectiveness and robustness have not met researchers' demand. Thus, several improvements of the method have been carried out in refs. [17, 21-24]. a-MFA was developed in [17] by applying modifications on distance of each two fireflies and on step parameter $\alpha$. In [21], KazemzadehParsi has proposed improved firefly algorithm (IFA) by applying three modifications. In [22], the memetic firefly algorithm (MFA) has been developed by balance of exploration acting as global search and exploitation acting as local search. In [23], FA with adaptive parameter a (ASPFA) was proposed by suggesting adaptive equation for updating a based on current and highest iterations. Quasi-chaos FA was developed in [24] by using Quasi-chaotic phenomenon and its performance was tested on different benchmark functions. In [16], Moustafa et al have run four methods consisting of FA, IFA, MFA and ASPFA on ELD problem with 3-unit system and 6-unit system. The final comparison has reported that IFA in [21] was the best method and FA was the worst method.However, the authors have not compared these methods with other existing methods to give more evidences for conclusion.

In the paper, we propose a new technique for generating new solutions of FA to construct an effectively modified FA (EMFA) for dealing with different study cases of ELD problem and comparing with existing FA methods and other existing methods. The new technique will choose the best way for newly updating each considered solution based on their quality. If a considered solution is close to good solution group with low fitness function, its new solution will be produced by searching nearby its current position. In case that the considered solution is far away the good solution group, its new solution is found nearby the current best solution. The new technique can determine a better search zone for each considered solution in aim to fast converge to optimal solution and avoid falling into local optimal solutions. The effectiveness of the EMFA method is investigated by testing on different systems with different constraints. Consequently, in the paper our main contributions are as follows:

a. Point out disadvantages of conventional FA and propose modifications for improving performance

b. Demonstrate advantages of the proposed method over other improved versions of FA, which are available in other studies

c. Contribute a potential method for ELD problem

\section{Problem Formulation}

\subsection{Objective Function}

In ELD with single fuel, the fuel cost of each generating unit is expressed as a quadratic function of its power output. The objective of the problem is to minimize the total fuel cost of $N$ available units and can be written as follows:

$$
\operatorname{Min} F=\sum_{s=1}^{N} F_{s}\left(P_{s}\right),
$$

where $P_{s}$ is real power output of generator $s ; F_{s}$ is the fuel cost function of thermal unit $s$ and it can be represented in equation 2 and equation 3 corresponding to single fuel and multi fuel cases [4].

$$
\begin{aligned}
& F_{s}\left(P_{s}\right)=a_{s}+b_{s} P_{s}+c_{s} P_{s}^{2}(\$ / \mathrm{h}), \\
& F_{s}\left(P_{s}\right)=\left\{\begin{array}{l}
a_{s 1}+b_{s 1} P_{s}+c_{s 1} P_{s}^{2}, \text { fuel } 1, P_{s, \text { min }} \leq P_{s} \leq P_{s 1, \text { max }} \\
\vdots \\
a_{s N F s}+b_{s N F s} P_{s}+c_{s N F s} P_{s}^{2}, \text { fuel } N F s, P_{s N F s, \text { min }} \leq P_{s} \leq P_{s, \text { max }}
\end{array}\right.
\end{aligned}
$$

where $a_{s}, b_{s}$, and $c_{s}$ are fuel cost coefficients of unit $s$ with single fuel option; $P_{s, \min }$ and $P_{s, \max }$ represent the lowest value and the highest generations; $N F_{s}$ represents the number of fuel options of thermal unit $s$. 


\subsection{Set of Constraints}

Active power balance: Total energies generated by all generating units must satisfy electricity load and power losses dropped in transmission lines

$$
\sum_{s=1}^{N} P_{s}=P D+P \text { Loss },
$$

where $P D$ is demanded power by load; PLoss is power loss obtained by [1]:

$$
\text { PLoss }=\sum_{i=1}^{N} \sum_{j=1}^{N} P_{i} B_{i j} P_{j}+\sum_{i=1}^{N} B_{0 i} P_{i}+B_{00},
$$

where $B_{i j}, B_{0 i}, B_{00}$ are the coefficients of power loss matrix. Limitations of thermal generating units: Power output of each thermal generating unit must follow the rule.

$$
P_{s, \text { min }} \leq P_{s} \leq P_{s, \text { max }}
$$

\section{Proposed Method}

\subsection{Conventional Firefly Algorithm}

A firefly $i$ is also a solution $i$, which is represented as a position $X_{i}$ of firefly $i$. Each firefly $i$ will fly to other fireflies with better brightness. Thus, there is a possibility that each solution $i$ will own different positions $X_{i j}$ and the equation to determine $X_{i j}$ for firefly $i$ is obtained as follows:

$$
X_{i j}^{\text {new }}=X_{i}+\beta . \Delta X_{i j}+\text { rand }_{i},
$$

where $\Delta X_{i j}$ and $\beta$ are step size and the attractiveness of firefly $i$ calculated by:

$$
\begin{aligned}
& \Delta X_{i j}=\left(X_{j}-X_{i}\right) \\
& \beta=\beta_{0} e^{-\gamma R_{i j}^{2}},
\end{aligned}
$$

Where $\beta_{0}$ is initial attractiveness; and $R_{i j}$ is the distance between fireflies $i$ and $j$ obtained by:

$$
R_{\mathrm{ij}}=\sqrt{\left(X_{i}-X_{j}\right)^{2}} .
$$

The whole search procedure of FA can be described as the following steps:

Step 1: Enter necessary data of optimization problems

Step 2: Select maximum iteration $(G)$ and population $\left(N_{F}\right)$, and produce population randomly Step 3: Calculate fitness and determine the best solution Gbest. Set current iteration $(C l)$ to 1

Step 4: Start solution $X_{i}(i=1)$

Step 5: Generate $X_{\text {inew }}$ by using (7)-(10)

Step 6: Calculate fitness function of $X_{\text {innew }}$

Step 7: Set $X_{\text {innew }}$ with the best fitness function to $X_{\text {inew }}$

Step 8: If $i=N_{F}$, go to next step. Otherwise, set $i=i+1$ and back to step 5 .

Step 9: Compare $X_{i}$ and $X_{\text {inew }}$ to keep better one. Determine the best solution Gbest

Step 10: If $\mathrm{Cl}$ is equal to $\mathrm{G}$, stop the search process. Otherwise, set $\mathrm{Cl}=\mathrm{Cl}+1$ and back to step 4.

\subsection{The Proposed EMFA Method}

In the proposed method, we propose a new technique to generate updated step size and new solutions. At the beginning, $\Delta F F_{i}$ and $\Delta F F_{\text {mean }}$ are determined by the following equations: 


$$
\begin{aligned}
& \Delta F F_{i}=\frac{F F_{i}-F F_{\text {Best }}}{F F_{\text {Best }}} \\
& \Delta F F_{\text {mean }}=\frac{F F_{\text {mean }}-F F_{\text {Best }}}{F F_{\text {Best }}}
\end{aligned}
$$

Where $F F_{i}, F F_{\text {Best }}$ and $F F_{\text {mean }}$ are the fitness function of solution $i$, the best solution and the population on average. Corresponding to the result of comparison between $\triangle F F_{i}$ and $\Delta F F_{\text {mean, }}$, the updated step size is calculated and then the new solutions of $X_{i}$ can be determined. As a result, the new proposed technique is described in detail as below

$$
\begin{gathered}
\text { If } \Delta F F_{i}<\Delta F F_{\text {mean }} \\
\Delta X_{i j}=X_{j}-X_{i}
\end{gathered}
$$

else

$$
\begin{aligned}
& \Delta X_{i j}=\left(X_{j}-X_{i}+X_{r 1}-X_{r 2}\right) \\
& X_{\text {ijnew }}=\text { Gbest }+ \text { randn. } \beta . \Delta X_{i j}+\text { randn, } \% \mathrm{X}_{\mathrm{r} 1} \text { and } X_{\mathrm{r} 2} \text { are two randomly chosen } \\
& \text { solutions }
\end{aligned}
$$

End

The whole search process of the proposed method is also implemented similarly to that of FA; however, there is only one difference of the two methods that is the new solution generation procedure.

\section{The Implementation of EMFA for ELD Problem}

\subsection{Selecting Decision Variables and Handling Active Power Balance Constraint}

Each solution or each position of firefly $i$ contains active power of $(N-1)$ thermal units. Solution $X_{i}$ is mathematical modeled in Equation 17.

$$
X_{i}=\left[P_{2, i}, P_{2, i}, \ldots, P_{N, i}\right] ; i=1, \ldots, N_{F}
$$

Furthermore, $X_{i}$ must always meet the following rule:

$$
X_{\min } \leq X_{i} \leq X_{\max },
$$

Where $X_{\min }=\left[P_{2, \min }, P_{3, \min ,}, \ldots, P_{N, \min }\right]$ and $X_{\max }=\left[P_{2, \max }, P_{3, \max }, \ldots, P_{N, \max }\right]$

Finally, power output of the first thermal unit is calculated by [12]:

$$
P_{1, i}=P D+P \operatorname{Loss}-\sum_{s=2}^{N} P_{s, i} .
$$

\subsection{Fitness Function}

Fitness function of each solution must be obtained for evaluating the quality of solution. Generally, the function is composed of objective function and punishment of the violation of dependent variables. For ELD problem, it is established as follows:

$$
F F_{i}=\sum_{s=1}^{N} F_{s}\left(P_{s}\right)+P F \times\left(\text { Punishment }_{i}\right)^{2}
$$


where PF is a factor; and Punishment $i$ is the violation punishment determined by:

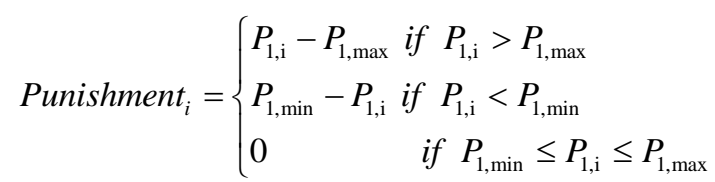

\section{Numerical Results}

The proposed EMFA is tested on four systems and obtained results are compared to FA methods and other existing methods. The detail of four test systems is described as follows: Case 1: Six-unit test system neglecting and considering power losses Case 2: Six-unit test system considering prohibited working zones and power losses Case 3: Twenty-unit test system considering power losses

Case 4: Ten-units with multi fuels and four load cases of 2400, 2500, 2600 and 2700 MW corresponding to cases $4.1,4.2,4.3$ and 4.4 .

For each study case, EMFA is run fifty independent trials by using Matlab and computer with $4 \mathrm{~GB}$ of Ram and $2.4 \mathrm{Ghz}$ processor.

\subsection{Result Comparisons for the First System}

In the section, the proposed method is compared to other FA methods reported in [16]. For running the proposed method, population and maximum iteration are set to 10 and 30 for the proposed method while authors in [16] have set 25 and 150 for the two parameters for FA, IFA, ASPFA and MFA. Table 1 and Table 2 show the comparison for different loads with the case of neglecting and considering power losses, respectively. The comparison can see that the proposed method can reach less cost than FA, IFA, ASPFA and MFA by $\$ 43.37, \$ 1.37$, $\$ 130.37$ and $\$ 35.37$ for $600 \mathrm{MW}$ load. Equally, the proposed method also reaches better cost than other methods for other cases. As a result, it can lead to a conclusion that the proposed method is more potential than other FA methods in terms of less population, less iteration and better optimal solutions.

Table 1. The Best Cost Comparisons $(\$ / h)$ for the First System without Power Losses

\begin{tabular}{cccccc}
\hline PD & FA & IFA & ASPFA & MFA & EMFA \\
$(\mathrm{MW})$ & {$[16]$} & {$[16]$} & {$[16]$} & {$[16]$} & \\
\hline 600 & 31489 & 31447 & 31576 & 31481 & 31445.62 \\
700 & 36075 & 36006 & 36036 & 36021 & 36003.12 \\
800 & 40739 & 40676 & 40701 & 40740 & 40675.97 \\
\hline
\end{tabular}

Table 2. The Best Cost Comparisons ( $\$ / h)$ for the First System with Power Losses

\begin{tabular}{cccccc}
\hline PD (MW) & FA[16] & IFA [16] & ASPFA [16] & MFA [16] & EMFA \\
\hline 600 & 32122 & 32098 & 32159 & 32109 & 32094.7 \\
700 & 37004 & 36914 & 36960 & 36978 & 36912.1 \\
800 & 41939 & 41898 & 41976 & 41930 & 41896.6 \\
\hline
\end{tabular}

\subsection{Result Comparisons for the Second System}

For the system with POZ and power loss constraint, the proposed method is compared to FA and other methods. The results in terms of the best cost, mean cost and the worst cost, and the control parameters consisting of $N_{F}$ and $G$ are also given in Table 3 . It is surprised to note that the minimum cost of $\alpha$-MFA is the best value among the reported methods but the real cost, which is recalculated, is $\$ 15443.23$. Clearly, the accuracy of the number is not confirmed certainly. In comparisons with FA, we have set its maximum iteration to 60 while that of the proposed method has been set to 15; however, all the costs of the proposed method were less than those of FA. Clearly, the proposed method could reach better results than FA including optimal solutions and convergence speed.

For comparison with other methods, the proposed method has reached the best results with lower best cost, lower mean cost and lower worst cost meanwhile it has used the lowest 
population and the lowest maximum iteration. In summary, the proposed method is much better than FA and other methods regarding optimal solution, convergence speed and stable search ability for the system with $\mathrm{POZ}$ and power loss constraints.

Table 3. Result Comparisons for System 2

\begin{tabular}{ccccc}
\hline Method & $\begin{array}{c}\text { Best cost } \\
(\$ / \mathrm{h})\end{array}$ & $\begin{array}{c}\text { Mean cost } \\
(\$ / \mathrm{h})\end{array}$ & $\begin{array}{c}\text { Worst } \\
\operatorname{cost}(\$ / \mathrm{h})\end{array}$ & $N_{F} \times G$ \\
\hline DE[5] & $15,449.77$ & $15,449.87$ & $15,449.78$ & $36 \times 100$ \\
GA [5] & 15,459 & 15,524 & 15,469 & $36 \times 100$ \\
PSO [5] & 15,450 & 15,492 & 15,454 & $36 \times 100$ \\
KHA1 [19] & $15,450.75$ & $15,452.82$ & $15,455.50$ & $50 \times 100$ \\
KHA2 [19] & $15,448.21$ & $15,450.83$ & $15,453.40$ & $50 \times 100$ \\
KHA3 [19] & $15,445.36$ & $15,447.22$ & $15,449.60$ & $50 \times 100$ \\
KHA4 [19] & $15,443.08$ & $15,443.19$ & $15,443.30$ & $50 \times 100$ \\
a-MFA[17] & 15442.9 & - & - & $12 \times 100$ \\
FA & 15445.087 & 15479.378 & 15575. & $10 \times 60$ \\
EMFA & 15443.075 & 15443.077 & 15443.12 & $10 \times 15$ \\
\hline
\end{tabular}

\subsection{Result Comparison for the Third System}

In this section, FA and the proposed method are run on a test system with 20 unit considering power losses. In Table 4, result reported is only the best cost while other costs such as mean cost and maximum cost are neglected because most compared methods have not shown these values. In fact, only improved CSA (ICSA) [12] reported all values and its standard deviation cost is approximately equal to zero but its maximum iteration is 500 while that of the proposed method is 200. As we have increased the maximum iteration to 500 , the standard deviation of the proposed method was also zero. In comparisons with other methods, it can reveal that the proposed method can also obtain approximate solution quality with CSA, ORCSA and better solution quality than BBO and FA; however, the result of $\left(N_{F} x G\right)$ of the proposed method is much less than that of other ones. Clearly, the proposed method is more effective and faster than FA and other methods for the case.

Table 4. Result Comparisons for System 3

\begin{tabular}{ccc}
\hline Method & Best Cost $(\$ / h)$ & $N_{F} \times G$ \\
\hline CSA [12] & 62456.633 & $10 \times 500$ \\
ICSA [12] & 62456.633 & $10 \times 500$ \\
BBO [14] & 62456.7926 & $50 \times 400$ \\
FA & 62458.881 & $10 \times 1000$ \\
EMFA & 62456.633 & $10 \times 200$ \\
\hline
\end{tabular}

\subsection{Result Comparisons for the Fourth System}

In this section, FA and the proposed method are run on a test system with ten units using multi fuels. Four loads of $2400,2500,2600$ and $2700 \mathrm{MW}$ are employed in turn. For the system, population of FA and the proposed method are set to 10 but the maximum iteration of FA is set to 300 whereas that is set to 50 for the proposed method. Clearly, the purpose of the selection aims to demonstrate the outstanding convergence speed of the proposed method over FA. As a result, the best cost, the population and the maximum iteration from FA, the proposed method and other methods are reported in Table 5 . It is clear that the proposed method has found better cost than FA and approximate cost with other methods but the result of $\left(N_{F} X G\right)$ of the proposed method is much less than that of FA and other methods. The comparisons can result in a conclusion that the proposed method has the same solution quality with other methods but reaches the fast convergence speed. 
Table 5. The Result Comparison for System 4 with Different Loads

\begin{tabular}{cccccc}
\hline Method & 2400 & 2500 & 2600 & 2700 & \multirow{2}{*}{$N_{F} \times G$} \\
\hline HRCGA [4] & 481.723 & 526.238 & 574.3808 & 623.809 & $10 \times 800$ \\
RCGA [4] & 481.723 & 526.239 & 574.3966 & 623.809 & $10 \times 800$ \\
DE [5] & 481.723 & 526.239 & 574.381 & 623.809 & $60 \times 200$ \\
IEP [6] & 481.779 & 526.304 & 574.473 & 623.851 & - \\
CA [8] & 481.723 & 526.24 & 574.381 & 623.809 & $30 \times 100$ \\
HICDE [7] & 481.723 & 526.239 & 574.381 & 623.809 & $20 \times 200$ \\
FA & 485.6 & 528.1 & 577.0 & 627.89 & $10 \times 300$ \\
EMFA & 481.723 & 526.238 & 574.381 & 623.809 & $10 \times 50$ \\
\hline
\end{tabular}

\section{Conclusion}

In this paper, an effectively modified firefly algorithm has been applied for solving four systems of ELD problem. In the four systems, different types of fuel cost function such as single fuel and multi fuels, and different constraints such as power losses and prohibited working zones are taken into account in aim to test the performance of the proposed method. The proposed method has used a new technique for searching new solutions that FA has coped with low quality solutions and low convergence. The result comparison can lead to a conclusion that the proposed method is much better than FA in terms of finding higher quality solution, using lower population and lower maximum iteration. Comparing the proposed method with other existing methods also results in the same evaluation that the proposed method can obtain better or approximate quality solution with faster convergence speed. Consequently, the proposed method is an effective and robust method for dealing with ELD problem.

\section{References}

[1] Rao DSNM, Kumar, N. Comparisional Investigation of Load Dispatch Solutions with TLBO. International Journal of Electrical and Computer Engineering (IJECE). 2017; 7(6): 3246-3253.

[2] Yasin ZM, Aziz NFA, Salim NA, Wahab NA, Rahmat NA. Optimal Economic Load Dispatch using Multiobjective Cuckoo Search Algorithm. Indonesian Journal of Electrical Engineering and Computer Science (IJEECS). 2018; 12(1):168-174.

[3] Karthik N, Parvathy AK, Arul R. Non-convex Economic Load Dispatch using Cuckoo Search Algorithm. Indonesian Journal of Electrical Engineering and Computer Science (IJEECS). 2017; 5(1): 48-57.

[4] Baskar S, Subbaraj P, Rao MVC. Hybrid real coded genetic algorithm solution to economic dispatch problem. Computers \& Electrical Engineering. 2003; 29(3): 407-419.

[5] Noman N, Iba H. Differential evolution for economic load dispatch problems. Electric Power Systems Research. 2008; 78(8): 1322-1331.

[6] Park YM, Won JR, Park JB. A new approach to economic load dispatch based on improved evolutionary programming. Engineering Intelligent Systems for Electrical Engineering and Communications. 1998; 6(2): 103-110.

[7] Panigrahi BK, Yadav SR, Agrawal S, Tiwari MK. A clonal algorithm to solve economic load dispatch. Electric Power Systems Research. 2007; 77(10): 1381-1389.

[8] Balamurugan R, Subramanian S. Hybrid integer coded differential evolution-dynamic programming approach for economic load dispatch with multiple fuel options. Energy Conversion and Management. 2008; 49(4): 608-614.

[9] Bard JF. Short-term scheduling of thermal-electric generators using Lagrangian relaxation. Operations Research. 1998; 36(5): 756-766.

[10] Su CT, Lin CT. New approach with a Hopfield modeling framework to economic dispatch. IEEE Transactions on Power Systems. 2000; 15(2): 541-545.

[11] Mustafa MW, Abdilahi AM, Mustapha M. Chaos-Enhanced Cuckoo Search for Economic Dispatch with Valve Point Effects. TELKOMNIKA (Telecommunication Computing Electronics and Control), 2016; 14(4): 1220-1227.

[12] Nguyen TT, Vo DN. The application of one rank cuckoo search algorithm for solving economic load dispatch problems. Applied Soft Computing. 2015; 37: 763-773.

[13] Gachhayat SK, Dash SK, Ray P. Multi Objective Directed Bee Colony Optimization for Economic Load Dispatch With Enhanced Power Demand and Valve Point Loading. International Journal of Electrical and Computer Engineering (IJECE). 2017; 7(5): 2382-2391.

[14] Bhattacharya A, Chattopadhyay PK. Biogeography-based optimization for different economic load dispatch problems. IEEE transactions on power systems. 2010; 25(2): 1064-1077. 
[15] Yu J, Kim CH, Wadood A, Khurshiad T, Rhee SB. A Novel Multi-Population Based Chaotic JAYA Algorithm with Application in Solving Economic Load Dispatch Problems. Energies. 2018; 11(8): $1-26$.

[16] Moustafa FS, El-Rafei A, Badra NM, Abdelaziz AY. Application and performance comparison of variants of the firefly algorithm to the economic load dispatch problem. In Advances in Electrical, Electronics, Information, Communication and Bio-Informatics (AEEICB), 2017 Third International Conference on. 2017; 147-151.

[17] Subramanian R, Thanushkodi K, Prakash A. An Efficient Meta Heuristic Algorithm to Solve Economic Load Dispatch Problems. Iranian Journal of Electrical and Electronic Engineering. 2013; 9(4): 246252.

[18] Shaw B, Ghoshal S, Mukherjee V, Ghoshal SP. Solution of economic load dispatch problems by a novel seeker optimization algorithm. International Journal on Electrical Engineering and Informatics (IJEEI). 2011, 3(1), 26-42.

[19] Mandal B, Roy PK, Mandal S. Economic load dispatch using krill herd algorithm. International journal of electrical power \& energy systems. 2014; 57: 1-10.

[20] Yang XS. Nature-inspired metaheuristic algorithms. 1st, Frome, UK: Luniver Press., 2008.

[21] Kazemzadeh-Parsi MJ. A modified firefly algorithm for engineering design optimization problems. Iranian Journal of Science and Technology. Transactions of Mechanical Engineering. 2014; 38(M2): 403.

[22] Fister Jr I, Yang XS, Fister I, Brest J. Memetic firefly algorithm for combinatorial optimization. arXiv preprint arXiv. 2012; 1204-5165.

[23] Yu S, Zhu S, Ma Y, Mao D. A variable step size firefly algorithm for numerical optimization. Applied Mathematics and Computation. 2015; 263: 214-220.

[24] Yu WX, Wang JN, Li Y, Wang ZH. The Chaos and Stability of Firefly Algorithm Adjacent Individual. TELKOMNIKA (Telecommunication Computing Electronics and Control). 2017; 15(4): 1733-1740. 